Future considerations for the mRNA-lipid nanoparticle vaccine platform

Botond Z. Igyártó*, Sonya Jacobsen and Sonia Ndeupen

Thomas Jefferson University, Department of Microbiology and Immunology, 233

South 10 ${ }^{\text {th }}$ Street, Philadelphia, PA 19107.

Vaccines based on mRNA-containing lipid nanoparticles (LNPS) are a promising new vaccine platform used by two of the leading vaccine candidates against coronavirus disease in 2019 (COVID-19). However, there are many questions regarding their mechanism of action in humans that remain unanswered. Here we consider the immunological features of LNP components and off-target effects of the mRNA, both of which could increase the risk of side effects. We suggest ways to mitigate these potential risks by harnessing dendritic cell (DC) biology.

Keywords: mRNA-LNP vaccines, side effects, dendritic cells

Correspondence:

*e-mail: botond.igyarto@jefferson.edu 
COMMENT - During writing, keep this paragraph as it creates the drop required for the opening page and enables you to the write to fit. Leave space for a display item if applicable. Note that the heading Introduction is not in the layout and the affiliations, email and DOI are outside the text area, so these lines can be ignored when judging the length.

\section{Future considerations for the mRNA-lipid nanoparticle vaccine platform}

\section{Botond Z. Igyártó*, Sonya Jacobsen and Sonia Ndeupen}

Vaccines based on mRNA-containing lipid nanoparticles (LNPs) are a promising new vaccine platform used by two of the leading vaccine candidates against coronavirus disease in 2019 (COVID19). However, there are many questions regarding their mechanism of action in humans that remain unanswered. Here we consider the immunological features of LNP components and off-target effects of the mRNA, both of which could increase the risk of side effects. We suggest ways to mitigate these potential risks by harnessing dendritic cell (DC) biology.

LNPs have grown in popularity as a delivery and adjuvant system for mRNA vaccines. An abundance of animal studies have shown the promise of this platform ${ }^{1}$ and human clinical trials by Moderna and BioNTech/Pfizer of mRNA-LNP-based SARS-CoV-2 vaccines recently reported above $90 \%$ protection rates. The advantages of using LNPs for vaccines are numerous. In addition to being a safer alternative to viral vectors for the delivery of mRNA vaccines, LNPs are self-adjuvating and highly customizable. Furthermore, the LNP-mRNA platform can be manufactured on a large scale and adapted easily to emerging pathogens. Also, the recent development of thermostable variants ${ }^{2}$ will overcome the necessity of cold-chain storage, which is required to different degrees for the current mRNA-LNP-based SARS-CoV-2 vaccines. However, because this is a new approach for human vaccination, with different levels of reported side-effects $^{3}$, there remain many unknowns and caveats that should be considered.

\section{Immunological features of LNPs}

LNPs are $\sim 100 \mathrm{~nm}$ size carriers that consist of phospholipids, cholesterol, PEGylated lipids and cationic or ionizable lipids. The phospholipids and cholesterol have structural and stabilizing roles whereas the PEGylated lipids support prolonged circulation. Cationic lipids are included to allow complexation of the negatively charged mRNA molecules and to enable exit of the mRNA from the endosome to the cytosol for translation ${ }^{4}$. Although cationic lipid containing LNPs were considered by some to be immunologically inert ${ }^{5}$, other data support that many are indeed highly inflammatory and possibly cytotoxic ${ }^{4}$. Various cationic lipids have been shown to activate different inflammatory pathways and induce complement activation related pseudoallergy 4 . In concordance with these, we observe that empty LNPs $^{5}$ given intradermally to mice induce robust inflammatory responses, characterized by neutrophil infiltration, inflammatory cytokine production, activation of diverse inflammatory pathways and excessive cell death (unpublished observations). The presentation of self-antigens in this highly inflammatory environment might ultimately lead to a break in tolerance. Therefore, we believe more careful characterization of LNPs is needed, and suggest that only the inflammatory, but minimally cytotoxic lipids are approved as vaccine components (Figure 1).

Some DC subsets at optimized antigen dose can induce protective antibody responses in the absence of inflammatory agents ${ }^{6,7}$. These data suggest that LNP-based vaccine platforms that lack inflammatory cationic/ionizable lipids, could be a viable option to induce protective antibody responses if targeted to certain DC subsets.

\section{Off-target effects of vaccine mRNA}

Based on the current mRNA-LNP vaccine design, LNPs can be taken up by almost any cell type, near or far from the site of injection, transfecting them with the antigen-encoding mRNA ${ }^{8}$. Moreover, the mRNA used in these vaccines are modified to increase its stability in vivo, allowing extended periods of mRNA translation 9,10 . Also, a significant portion of the mRNA can be re-packaged and expelled from transfected cells in extracellular vesicles ${ }^{11}$. These vesicles could reach cells far from the injection site, further increasing the number of cells translating the antigen and extending the duration of its expression.

Long-term mRNA translation in non-professional APCs might lead to unanticipated cell killing. Similar to any other self-protein, synthesized vaccine proteins have access to antigen presentation on MHC class I molecules on any nucleated cells ${ }^{12}$. Thus, any cell presenting antigenic determinants from the 
vaccine could become a target of T cell-mediated killing. Furthermore, if vaccine-derived proteins become inserted into the plasma membrane or secreted and associated with cell membrane, these cells could become targets of antibody-dependent cellular cytotoxicity ${ }^{13}$. Both should become evident after an adaptive immune response has been generated and may be accentuated upon secondary immunization (Figure 2). In line with this, systemic adverse events from the mRNA-LNP based SARS-CoV-2 vaccines were indeed more common after the second vaccination, particularly with the highest dose ${ }^{3}$. Strategies that allow delivery of the mRNA exclusively to DCs with the use of DC-targeting antibodies or ligands may limit the possible off-target effects.

\section{Considering DC biology}

The success of mRNA-LNP vaccines depends not only on cellular internalization of the LNPs, but on the release of mRNA from the endosomal compartment, to enable translation. It is thought that, in most cells, the ionizable lipid component becomes protonated in the progressively acidic environment of the endosome, leading to endosome destabilization and RNA release. ${ }^{4}$ However, DCs have specific biology that may interfere with this process. Specifically, DCs have been reported to retain intact protein antigens for days ${ }^{7,14}$ in mildly acidic endosomal compartments ${ }^{14}$. This likely allows DCs more time to display antigenic determinants to T cells and intact antigens to B cells ${ }^{7}$. However, the low acidity environment of the DC endo-lysosomal compartment may inhibit endosomal escape of mRNA by failing to ionize the lipids in the LNPs. While it remains to be tested, lipid carriers that fuse with the plasma membrane and release their mRNA cargo into the cytosol might be preferred when it comes to aiding mRNA translation and subsequent antigen presentation in DCs.

\section{Considering pre-existing inflammation}

It has been shown that mRNA-LNP vaccines have an altered tissue distribution, dynamics and uptake in animals that have been pre-exposed to inflammatory agents ${ }^{4}$. These findings suggest that people with preexisting inflammatory conditions might show altered immune responses to these vaccines.

\section{Considering vaccine delivery route}

The route of vaccine delivery determines which tissue will be protected by the cellular immunity. Peripheral DCs programme antigen-specific T cells in the lymphoid organs to migrate to and reside in the DC's tissue of origin ${ }^{15}$. However, most current vaccines, including the mRNA-LNP based SARS-CoV-2 vaccines, are delivered via the skin or muscle. These delivery routes are expected to support the formation of antibodies that provide systemic protection and $\mathrm{T}$ cells that patrol these organs but not the site of natural exposure and infection, the airway epithelium. The presence of virus-specific T cells in the right tissue would be highly desirable because these cells can also provide cross-protection across different strains of viruses ${ }^{16}$. Therefore, we would propose tailoring the vaccine's route of administration to the pathogen's natural route of infection and developing intranasal vaccines for respiratory viruses such as influenza virus and SARS-CoV-2.

\section{Broadening vaccine-induced $T$ cell responses}

Our knowledge of immune mechanisms of mRNALNP vaccines is still very limited. Vaccine-derived mRNAs are expected to be translated and presented by MHC class I but largely excluded from MHC class II ${ }^{12}$. Yet, the existing mRNA-LNP vaccination studies clearly show that both CD8+ $\mathrm{T}$ cell and CD4+ $\mathrm{T}$ cell responses are induced ${ }^{1}$.

The type of Th cell response induced depends on the DC subsets and pattern-recognition receptor (PRR) pathways engaged ${ }^{17}$. So far, the mRNA-LNP platform has been reported to induce Th1 and T follicular helper cells, likely through engagement of TLRs by cationic lipids 4 . To induce other Th cell subsets with the mRNA-LNP platform, we propose two strategies. First, PRR ligands could be included in the LNPs and, second, mRNAs encoding T cell-polarizing cytokines could be added to the LNPs. The first option is more restrictive as not all DC subsets express the same PRR repertoire, and thus not all DC subsets will be able to respond to the stimuli carried by the LNPs. Delivering mRNAs encoding polarizing cytokines would overcome this problem and would allow any DC subset, independent of its PRR profile, to polarize naive CD4+ T cells towards the desired lineage. Thus, with mRNA technology, there is almost no limit to modifying DC biology to match our needs.

\section{References}

1. Alameh, M.-G., Weissman, D. \& Pardi, N. Messenger RNA-Based Vaccines Against Infectious Diseases. in (2020). doi:10.1007/82 2020202

2. Zhang, N. N. et al. A Thermostable mRNA Vaccine against COVID-19. Cell (2020). doi:10.1016/j.cell.2020.07.024 Jackson, L. A. et al. An mRNA Vaccine against SARS-CoV-2 Preliminary Report. N. Engl. J. Med. (2020). doi:10.1056/nejmoa2022483

Samaridou, E., Heyes, J. \& Lutwyche, P. Lipid nanoparticles for nucleic acid delivery: Current perspectives. Advanced Drug Delivery Reviews (2020). doi:10.1016/j.addr.2020.06.002

Pardi, N. et al. Nucleoside-modified mRNA vaccines induce potent T follicular helper and germinal center B cell responses. J. Exp. Med. 215, 1571-1588 (2018)

Yao, C. et al. Skin dendritic cells induce follicular helper T cells and protective humoral immune responses. J. Allergy Clin. Immunol. (2015).

Bouteau, A. et al. DC subsets regulate humoral immune responses by supporting the differentiation of distinct TFH cells. Front. Immunol. (2019). doi:10.3389/fimmu. 2019.01134

Pardi, N. et al. Expression kinetics of nucleoside-modified mRNA delivered in lipid nanoparticles to mice by various routes. J. Control. Release 217, 345-351 (2015)

Karikó, K. et al. Incorporation of pseudouridine into mRNA yields superior nonimmunogenic vector with increased translational capacity and biological stability. Mol. Ther. (2008). doi:10.1038/mt.2008.200

$10 . \quad$ Karikó, K., Muramatsu, H., Ludwig, J. \& Weissman, D. Generating the optimal mRNA for therapy: HPLC purification eliminates immune activation and improves translation of nucleoside-modified, proteinencoding mRNA. Nucleic Acids Res. (2011). doi:10.1093/nar/gkr695

11. Maugeri, M. et al. Linkage between endosomal escape of LNP-mRNA and loading into EVs for transport to other cells. Nat. Commun. (2019). doi:10.1038/s41467-019-12275-6

12. Blum, J. S., Wearsch, P. A. \& Cresswell, P. Pathways of antigen processing. Annual Review of Immunology (2013).

processing. Annual Review of Immunology (2013). Gómez Román, V. R., Murray, J. C. \& Weiner, L. M. AntibodyDependent Cellular Cytotoxicity (ADCC). in Antibody Fc: Linking Adaptive and Innate Immunity (2013). doi:10.1016/B978-0-12- 
394802-1.00001-7
14. Lutz, M. B. et al. Intracellular routes and selective retention of antigens in mildly acidic cathepsin D/lysosome-associated membrane protein-1/MHC class II-positive vesicles in immature dendritic cells. J. Immunol 159, 3707-3716 (1997).

15. Sigmundsdottir, H. \& Butcher, E. C. Environmental cues, dendritic cells and the programming of tissue-selective lymphocyte trafficking. Nature Immunology (2008). doi:10.1038/ni.f.208

16. Clemens, E. B., Van de Sandt, C., Wong, S. S., Wakim, L. M. \& Valkenburg, S. A. Harnessing the power of T cells: The promising hope for a universal influenza vaccine. Vaccines (2018). hope for a universal influenza vacc
doi:10.3390/vaccines6020018

17. Janeway, C. A. \& Medzhitov, R. Innate immune recognition. Annu. Rev. Immunol. 20, 197-216 (2002).

Acknowledgements

This work was supported by NIAID R01AI146420 to B.Z.I.

${ }^{1}$ Department of Microbiology and Immunology, Thomas Jefferson University, Philadelphia, PA, USA. *e-mail: botond.igyarto@jefferson.edu

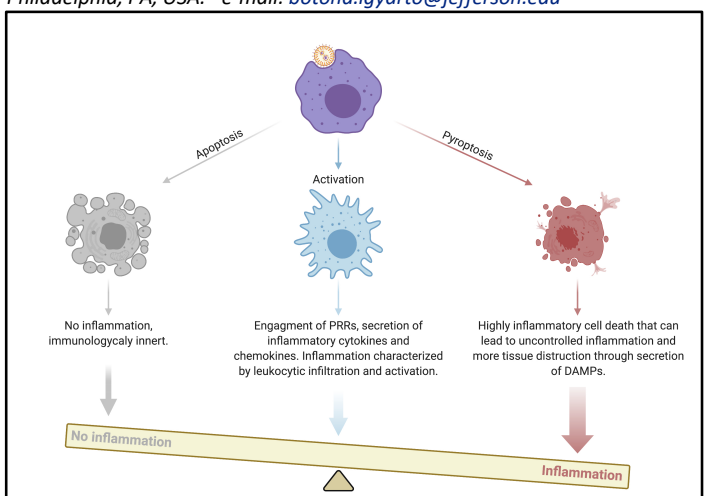

Figure 1. LNPs containing certain cationic/ionizable lipids are highly inflammatory. LNPS have been described to induce apoptosis, cell activation and death through engagements of PRRs and activation of inflammasomes. Excess inflammatory cell death in conjunction of already discussed off-target effects of the mRNA-LNP vaccines, could make this platform prone to support autoimmunity, high tissue destruction with serious side-effects. It will be necessary to strike a balance between non-inflammatory and highly inflammatory in order to induce a level of cell activation that is ideal for induction of protective immune responses.

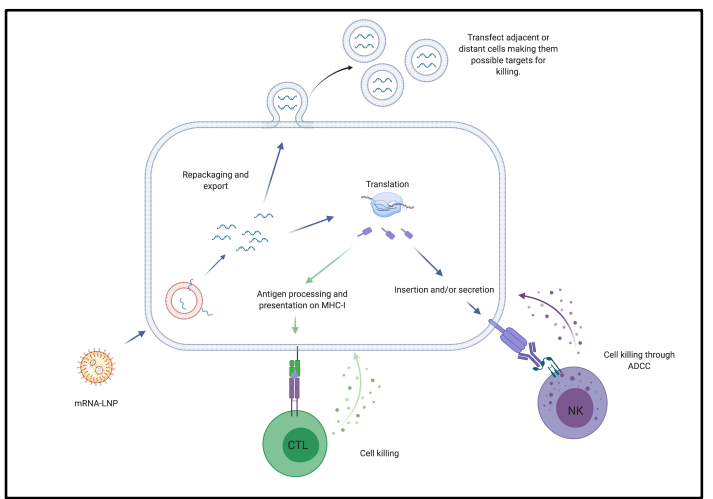

Figure 2. Possible off-target effects of the mRNA-LNP platform. The mRNA reaching the cytosol can have different fates. They can be re-packaged in EVs that can transfect adjacent or distant cells, making them target of the immune response induced by the vaccine. The intact antigen coded by the MRNA can in theory reach the plasma membrane marking the cells for killing through $A D C C$. The ADCC should become evident after the antibodies specific to this antigen are formed. Since all the nucleated cells express $\mathrm{MHC}-\mathrm{I}$, the translated protein can be processed and presented, as any other self-protein, on MHC-I to CD8 T cells. This can lead to cell killing after the effector $T$ cells are formed. The killing should be accentuated upon booster shot when the tissue memory $T$ cells are also present. 